Meta

Journal des traducteurs

Translators' Journal

\title{
Analyse du processus de la traduction : conséquences méthodologiques
}

\section{Nestor Schumacher}

Volume 18, numéro 3, septembre 1973

URI : https://id.erudit.org/iderudit/004058ar

DOI : https://doi.org/10.7202/004058ar

Aller au sommaire du numéro

Éditeur(s)

Les Presses de l'Université de Montréal

ISSN

0026-0452 (imprimé)

1492-1421 (numérique)

Découvrir la revue

Citer cet article

Schumacher, N. (1973). Analyse du processus de la traduction : conséquences méthodologiques. Meta, 18(3), 308-314. https://doi.org/10.7202/004058ar d'utilisation que vous pouvez consulter en ligne.

https://apropos.erudit.org/fr/usagers/politique-dutilisation/ 


\section{Analyse du processus de la traduction: conséquences méthodologiques}

Il semble que, dans le vaste domaine de l'étude de la traduction, l'intérêt se concentre de plus en plus sur l'analyse du processus lui-même. On trouve des preuves de cet intérêt dans des publications récentes comme l'ouvrage de Katharina Reiss : Möglichkeiten und Grenzen der Übersetzungskritik ${ }^{1}$, ou celui de R. Van den Broeck : Inleiding tot de vertaalwetenschap ${ }^{2}$. Rappelons également la journée de «Talf's Friends » (17 mars 1972) consacrée à la méthodologie de la traduction et à laquelle un praticien, F. Bovy, présentait un syllabus intitulé \& Processus et méthodologie de la traduction $»$. Tout récemment, au $3^{\text {e }}$ Congrès international de linguistique appliquée (Copenhague, 21-26 août 1972), les exposés fondamentaux du professeur A. Neubert ${ }^{3}$, de Leipzig, et de J. S. Holmes ${ }^{4}$, d'Amsterdam, manifestaient également cette préoccupation, Neubert affirmant, par exemple : «Dieser Prozess, als dessen Input der Quellentext T1 und als Output der Zieltext T2 stehen, ist der eigentliche schwarze Kasten, die «black box»der Übersetzungswissenschaft.»

Nous voudrions, pour notre modeste part, en nous fondant sur les remarques de ces chercheurs et sur notre expérience personnelle, proposer une analyse du processus de la traduction et en tirer quelques conclusions pratiques pour l'enseignement de celle-ci.

La traduction consistant dans la transposition d'un texte d'une langue, dite langue de départ, en une langue, dite langue d'arrivée - qui sera conçue ici comme étant 1a langue maternelle du traducteur - l'analyste est nécessairement amené à distinguer non pas deux, mais trois phases essentielles dans le processus. Nous appellerons ces phases : 1) phase d'assimilation, 2) phase de confrontation, 3) phase de restitution.

\footnotetext{
1. Munich, Max Hueber Verlag, 1971.

2. Louvain, Acco, 1972.

3. "Theorie und Praxis für die Ubersetzungswissenschaft ».

4. "The Name and Nature of Translation Studies".
} 


\section{PHASE D'ASSIMILATION}

Cette phase commence par un premier contact avec le texte, opération que certains auteurs appellent «appréhension» pour la distinguer de l'opération de «compréhension ». C'est en fait une simple lecture du texte dans son intégralité - insistons sur ce point trop souvent négligé par les traducteurs pressés. De ce premier contact résulte une double réaction dans l'esprit du traducteur.

\section{A. Première réaction}

La première réaction est une opération d'identification du texte, identification d'autant plus précise que le texte présente des caractéristiques plus typiques.

On a déjà proposé différentes classifications de textes du point de vue de la traduction, qui font en tout cas la distinction entre textes littéraires et textes non littéraires. K. Reiss propose une typologie plus complète, reprise d'ailleurs par Van den Broeck dans Inleiding tot de vertaalwetenschap. Neubert va encore plus loin et prévoit un dictionary of textual types ${ }^{5}$. Se fondant sur les fonctions essentielles de la langue, Reiss distingue :

a) Les textes où l'accent est mis sur le contenu (inhaltsbetont) et que l'on pourrait appeler avec Van den Broeck «référentiels» ou informatifs. Nous retrouverons ici tous les textes scientifiques et techniques, par exemple, le mode d'emploi d'un appareil ;

b) Les textes où l'accent est mis sur la forme (formbetont) et que l'on peut appeler expressifs. Ce seront tous les textes littéraires, pour lesquels le langage n'est pas seulement un moyen, mais un but ;

c) Les textes où l'accent est mis sur l'appel au lecteur (appellbetont) qui visent à le convaincre et que, faute d'un meilleur terme, nous appellerons exhortatifs, comme les textes publicitaires, les textes de propagande, notamment politiques.

Pour des raisons qui ne tiennent plus aux fonctions du langage, Reiss ajoute un quatrième type :

d) Le type audio-visuel, c'est-à-dire les textes qui ne parviennent au destinataire, en l'occurrence l'auditeur ou le spectateur, que par le canal de media tels que le théâtre, le cinéma, la radio, la télévision.

Nous préférons cette appellation à celle de «subsidiaire» de R. Van den Broeck, qui nous semble par ailleurs élargir abusivement cette notion en y incluant tous les textes religieux parce que composants d'un ensemble qui serait le culte. Un texte religieux peut être 1) informatif : par exemple, une mise au point sur une question de dogme ; 2) expressif : un psaume ; 3 ) exhortatif : une homélie ; 4) audio-médial : un Jeu de la Nativité. Cette remarque vise surtout à souligner la distinction entre type fonctionnel et catégorie de textes (textes religieux, juridiques, etc.).

L'identification du texte au point de vue typologique entraînera des différences fondamentales dans la manière de le traduire. En effet, le texte informatif

5. A. Neubert, "Invariance and Textual Types in Translation $" 3^{*}$ Congrès international de linguistique appliquée, Copenhague, 21-26 août 1972. 
fait référence à une réalité extra-linguistique qu'il s'agit simplement de communiquer le plus fidèlement possible au lecteur; le texte expressif propose une nouvelle réalité, l'œuvre en elle-même, qu'il faudra «re-créer » dans une langue pour laquelle elle n'a pas été conçue; le texte exhortatif - tout en pouvant participer par certains de ses éléments aux types précités - est essentiellement tourné vers le récepteur, chez lequel il s'agit de: produire le même effet; enfin le texte audiomédial est lié à certains moyens de communication bien définis et n'est qu'une composante — la seule à traduire — d'un ensemble immédiatement perceptible pour l'auditeur/spectateur allophone.

\section{B. Seconde réaction}

La seconde réaction suscitée dans l'esprit du traducteur par le contact avec le texte à traduire est la mise en branle de tout un background intellectuel et culturel. Automatiquement, le traducteur replace le texte dans un certain environnement, car par ses études, ses lectures, le cas échéant, des traductions antérieures, il a une certaine connaissance du domaine traité. Ceci met à sa disposition une série d'informations qui ne sont pas nécessairement: fournies par le texte lui-même : - informations extra-linguistiques sur le secteur de la science ou de la culture dont le texte relève ; - informations linguistiques : terminologie et éventuellement phraséologie en usage dans un secteur particulier.

Suite à sa première lecture, le traducteur a identifié le texte et déjà choisi l'orientation générale de sa traduction selon le type fonctionnel; il a situé le texte dans le cadre général de ses connaissances; il en a compris la substance. Il est même possible que, le texte ne dépassant pas sa compétence, il en ait déjà saisi tous les éléments. Dans ce cas, il peut passer à la phase suivante. Mais, dans la plupart des cas, le texte offre une certaine résistance. Certains passages restent obscurs et risquent de masquer l'un ou l'autre enchaînement. Un mot inconnu ou mal compris peut mettre sur une fausse piste. C'est ici qu'il importe de procéder - une première fois - à ce que notré collègue $M$. Spencer appelait dans un exposé récent competence self-evaluation, c'est-à-dire «the point in every definable stage of any translation process at which the translator decides to seek assistance outside his own brain ». Cette aide extérieure, à laquelle le traducteur décide de recourir, peut prendre des formes diverses : consultation d'ouvrages de référence, de dictionnaires explicatifs ou traductifs, de terminologies, etc., voire consultation d'un expert pour des textes de haute spécialisation.

On pourrait légitimement douter de la possibilité de bien traduire de tels textes sans être soi-même un spécialiste de la question si l'on ne faisait sienne la distinction pertinente de F. Bovy entre «connaissance » et « compréhension 》:

L'opération de traduction s'opère sur un texte déjà rédigé par une personne compétente et ce que le traducteur n'est pas nécessairement à même de connaître, il est en mesure de le comprendre; il n'est par conséquent pas compétent pour rédiger d'initiative le texte qui lui est soumis pour traduction. Le traducteur recherche la cohérence et la corrélation avec le connu (c'est une erreur de croire qu'il faut tout connaître pour traduire) et avec le véritable (sources de référence) 6 .

6. Syllabus, cité plus haut, Journée pédagogique pour les Instituts belges de traduction; 17 mars 1972 , p. 2. 
L'aide extérieure pourrait cependant s'avérer inopérante si le traducteur ne procédait consciencieusement au double mouvement d'analyse et de synthèse que requiert la compréhension parfaite de tout texte, quel qu'il soit. En effet, seule l'idée générale peut faire comprendre le rôle de chaque idée particulière, et ce n'est que par la compréhension de chaque idée particulière que l'ensemble sera vraiment reconstitué. Dans ce double mouvement de la pensée, il importe d'accorder une attention toute spéciale à certains mots (parfois très apparents comme les conjonctions, parfois plus effacés comme certains adverbes) qui marquent les enchaînements d'idées. Une compréhension parfaite suppose aussi que rien n'ait été laissé de côté et que tous les éléments — si minimes soient-ils, du moins en apparence - prennent leur place dans l'ensemble.

Le critère objectif d'une bonne compréhension nous paraît en effet être la cohérence : cohérence interne, c'est-à-dire adéquation de toutes les parties à l'ensemble - cohérence externe, c'est-à-dire cohérence entre ce texte et ce que nous savons du domaine plus général dont il fait partie.

\section{PHASE DE CONFRONTATION}

Après avoir ainsi pris connaissance du texte à traduire, l'avoir identifié et situé, l'avoir compris dans son ensemble comme dans ses différents éléments, nous passons à la phase de confrontation active entre les ressources de la langue de départ et de la langue d'arrivée.

Cette confrontation ne se conçoit qu'au niveau des unités de traduction. Cette notion, importante pour la méthodologie de la traduction recouvre un fragment Le texte suffisamment petit pour être isolé et suffisamment grand pour constituer un bloc traduisible. Elle se situe donc dans la «parole» au sens saussurien du terme et non dans la «langue», comme pourrait le faire un mot pris isolément. L'esprit se fixe sur cette unité et cherche parmi les équivalents potentiels de la langue d'arrivée l'équivalent optimal, parcourant ainsi les possibilités de la «langue » pour ensuite retourner à la «parole ». Qu'est-ce qui détermine cette équivalence optimale? C'est avant tout le microcontexte immédiatement contrôlable autour de l'unité de traduction et qui nous paraît correspondre en général à un couple de phrases : la phrase considérée + la phrase précédente à laquelle elle est souvent reliée par une certaine relation logique. Mais c'est aussi le macrocontexte (paragraphe, chapitre, ouvrage), dont les instructions restent en mémoire ou peuvent être contrôlées. C'est enfin la situation (temps, lieu, locuteur, destinataire, milieu, spécialité...) qui est souvent déterminante dans ce choix.

L'examen des unités de traduction se fera dans tout texte sur trois plans : a) Lexical : l'opération la plus fréquente est évidemment la confrontation de synonymes dans les deux langues considérées. Le rapprochement de plages sémantiques plus ou moins parallèles — à l'aide éventuellement de dictionnaires analogiques - stimulera la faculté d'intuition ;

b) Grammatical : bien que les structures de la langue d'arrivée soient ici plus contraignantes, il n'est pas douteux qu'il existe souvent plusieurs possibilités et que la structure optimale n'est pas nécessairement la structure type se substituant normalement à la structure de la langue de départ. Pensons aux différents moyens de rendre une relation de cause à effet, où le choix sera dicté par des considéra- 
tions de fréquence, certes, mais aussi de style au sens large (légèreté, concision, clarté...) ;

c) Idiomatique : où le traducteur doit particulièrement se méfier de la propension à «calquer» le syntagme qu'il a sous les yeux et chercher avant tout une équivalence fonctionnelle.

Ces trois plans constituent la base de toute confrontation LD/LA. Mais dès que nous avons affaire à un texte qui n'est plus simplement informatif, il convient de faire intervenir un point de vue prioritaire.

Pour le texte dit «expressif», ce sera le point de vue stylistique au sens propre, style d'une époque, style d'une école littéraire, style d'un auteur ainsi que le niveau de langue. Deux tendances s'affrontent ici : soit rapprocher le style de la norme de la LA, soit au contraire, comme le demande entre autres Ortega y Gasset, conserver tout le dépaysement d'un style étranger jusqu'aux limites supportables par la correction dans la langue d'arrivée.

Dans le cas du texte dit «exhortatif», l'essentiel est l'effet à produire. Il faudra donc tenir compte avant tout de la psychologie des destinataires, du caractère spécifique et du niveau de leur culture - à moins naturellement que l'on ne traduise ce texte que pour information. Ces critères influenceront surtout le choix des termes, souvent laudatifs ou au contraire péjoratifs, et qui, par exemple, dans un discours politique, seront riches en allusions, en slogans, en jeux de mots, etc. L'exemple des textes publicitaires - auquel on peut rattacher celui des titres de films - montre qu'une grande liberté de traduction est nécessaire si l'on veut produire un effet analogue sur des locuteurs d'une autre communauté linguistique.

Enfin, dans le cas du texte "audio-médial », c'est un point de vue technique qui va imposer une certaine liberté par rapport au texte original. Dans certaines techniques, par exemple, dans le Hörspiel, le texte sera, avec le bruitage, le seul support de la communication et le critère de la compréhension phonétique et sémantique sera prioritaire. Dans d'autres techniques, par contre, le texte ne jouera qu'un rôle subsidiaire et devra tenir compte de tout l'apport des autres éléments de la communication.

\section{PHASE DE RESTITUTION}

La confrontation, que nous avons décrite à ses différents niveaux, aboutit à la mise par écrit ou à l'enregistrement d'un projet de traduction. Tous les traducteurs sont d'accord sur ce point, encore que des divergences importantes subsistent quant au stade où s'élabore ce projet. F. Bovy, par exemple, le situe à un stade très précoce, antérieur à la compréhension totale à laquelle il servirait de support.

Ce premier projet, qui sera encore très ou trop fort calqué sur le mode d'expression de la langue de départ, servira de support à la seconde opération de compréhension, autour de laquelle s'articulera la fonction de maturation : mise en place des séquences de l'idée et compréhension finale de l'idée globale et de son cheminement ?.

Pour notre part, nous serions plutôt porté à exiger la compréhension complète avant toute élaboration de projet, situant celui-ci à un stade assez avancé. Nous proposons la démarche concrète suivante :

7. Op. cit. 
a) Grâce aux « outils de travail », le traducteur a parfaitement compris le texte à traduire et a rassemblé — sur une simple liste — tous les éléments qui échappaient à sa compétence ;

b) Muni ainsi de tout le «matériel » nécessaire, il manipule mentalement les unités de traduction en les prenant aussi larges que possible ;

c) Il met par écrit ou enregistre un texte presque définitif.

Restent alors deux opérations qui, en principe relèvent du travail de réviseur : d) une lecture critique du projet au point de vue de sa cohérence interne. Les unités de traduction ayant été manipulées de façon presque autonomes, leur rapprochement fera découvrir des bavures, des redondances, des lacunes. Une lecture à haute voix est ici un procédé très utile, qui s'impose naturellement pour un texte de type audio-médial. Elle fera ressortir les anomalies du rythme et amènera à supprimer ou à ajouter librement certains éléments ;

$e)$ Enfin, une dernière confrontation avec l'original permet de vérifier la conformité globale avec celui-ci, particulièrement au point de vue de la tonalité générale.

Nous avons, pour les besoins de l'analyse, distingué nettement trois phases dans le processus de traduction et décrit un certain nombre d'opérations. Il est clair que dans la pratique ces différentes phases se confondent partiellement, allant même jusqu'à donner l'impression d'une opération instantanée dans la traduction à vue. Elles n'en existent pas moins et leur description nous suggère quelques réflexions d'ordre méthodologique, également inspirées par notre expérience de professeur. Nous distinguerons celles qui se rapportent à l'organisation des cours dans un institut de traduction de celles qui concernent la méthodologie même des cours de traduction.

\section{A. Organisation}

a) $\mathrm{Si}$, comme nous le croyons, la typologie des textes décrite par les auteurs cités commande l'attitude générale du traducteur vis-à-vis de son texte, il importe que les différents types de texte - et non seulement différentes catégories soient abordés au cours des études. La répartition peut se faire au niveau du département entre professeurs d'une même langue. Il va de soi que les dispositions personnelles et notamment l'expérience acquise par le professeur dans un domaine particulier doivent jouer un rôle déterminant dans la répartition. Les types «expressif » et «audio-médial » notamment exigent une certaine formation théorique et pratique et surtout une créativité langagière qui ne s'improvisent pas. Éventuellement, on pourrait recourir ici à la critique de traductions existantes.

$b$ ) En ce qui concerne le type informatif, qui représente la plus grande masse de traduction, il semble nécessaire qu'un certain nombre d'heures de licence soient consacrées à des textes qui demandent une recherche terminologique et phraséologique, par exemple, à des textes scientifiques. Une certaine coordination entre $1^{\text {re }}$ et $2^{\mathrm{e}}$ licences est souhaitable pour assurer la base notionnelle et terminologique indispensable si l'on veut aborder en fin d'études des textes scientifiques relativement difficiles. Ici se pose tout particulièrement le problème complexe de la 
documentation. Une collaboration poussée entre le centre de documentation de l'établissement et les titulaires de ces cours permettrait aux étudiants d'effectuer la recherche sous la direction du professeur. Il y a évidemment avantage à choisir des secteurs qui ont été traités par les bureaux de terminologie existants.

c) On constate souvent chez les candidats-traducteurs un repliement - bien compréhensible - sur la langue écrite. La perte de contact avec l'expression orale est certes regrettable en soi et peut aussi faire du tort à l'expression écrite. C'est pourquoi nous sommes partisan de la traduction orale pour certaines heures de cours et de l'utilisation de l'appareil à dicter. Entre autres avantages, celui-ci permet de gagner du temps, donc de traiter une matière plus vaste, d'activer les connaissances, de traiter des unités plus grandes, de procéder à des corrections rapides.

\section{B. Méthodologie}

Quant à la méthodologie interne des cours de traduction, l'analyse à laquelle nous avons procédé suggère les remarques suivantes :

a) Dans le cadre d'un type et d'une catégorie de textes, le professeur de traduction a intérêt à choisir une suite de textes portant sur le même sujet. Cette méthode présente des avantages évidents quant à la documentation à réunir, aux notions à expliquer, à la terminologie à rechercher. Il serait souhaitable que l'étudiant puisse prendre connaissance de cette suite de textes dès le départ, car ils s'éclairent mutuellement.

b) La préparation peut différer selon la nature du texte. Nous proposons les deux schémas suivants : pour un texte de caractère général, les étudiants rassemblent, à domicile ou en bibliothèque, les éléments qui manquent à leur compétence, le cours est consacré à l'importante phase de confrontation, les étudiants proposant leurs traductions, le professeur se bornant à des remarques ou proposant une traduction optimale et la justifiant, la traduction définitive se fait par écrit à domicile; pour des textes spécialisés, il semble préférable que la phase d'assimilation se passe au cours sous la direction du professeur qui éclairera les notions et fournira des références pour la terminologie, la préparation se concentre ensuite sur la phase de confrontation avec recours aux sources indiquées, la rédaction définitive se fait au cours suivant.

c) Dans la phase de confrontation réalisée au cours, le professeur indique les unités de traduction, fait ressortir les mots ou locutions qui marquent les enchaînements d'idées, fait varier oralement les différentes traductions potentielles. Il distingue les transpositions nécessitées par les structures lexicales et grammaticales de la langue d'arrivée de celles qu'un traducteur compétent choisit librement pour des raisons de style ou d'effet à produire. De même, il précise les modifications qui lui sont inspirées par le macrocontexte ou la situation.

Souhaitons en terminant que ces quelques remarques, dont nous reconnaissons le caractère provisoire, provoquent un dialogue fructueux avec nos collègues des instituts de traduction qui se trouvent confrontés avec les mêmes problèmes.

NeSTOR SCHUMACHER 